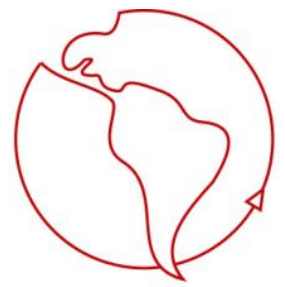

\title{
Pueblos Indígenas y Manejo de Recursos Hídricos en México
}

Francisco Peña

El Colegio de San Luis, México

frape@colsan.edu.mx

\section{Introducción}

Las comunidades indígenas de México con frecuencia han debido defender los recursos hídricos que necesitan para sostener sus formas de vida y subsistencia, frente a decisiones gubernamentales que no consideran sus necesidades y derechos. La desecación de lagunas y humedales, la inundación de pueblos y áreas agrícolas por la construcción de grandes presas, la contaminación de ríos y acuíferos por parte de la industria petrolera y la transferencia del agua a las grandes ciudades, son solo algunos ejemplos de las decisiones que han afectado a los pueblos indígenas en diversas regiones del país.

Los cambios registrados después de las elecciones federales del año 2000 no han significado modificaciones sustanciales para que los pueblos indígenas intervengan en la toma de decisiones para el manejo del agua, en particular de aquellas que significan alteraciones drásticas en los territorios que habitan, como es el caso de la construcción de grandes embalses para generar energía eléctrica.

Por el contrario, como veremos más adelante, existen datos de que el gobierno del presidente Vicente Fox está repitiendo el mismo tipo de medidas del pasado, en el campo de la construcción de presas contra el interés y derecho de las comunidades indígenas y campesinas ${ }^{1}$. El incumplimiento gubernamental de los acuerdos de San Andrés ${ }^{2}$, defendidos no solo por el zapatismo, sino también por la coalición más grande de organizaciones indígenas no armadas, prueba la posición adoptada por el gobierno federal en este campo.

Estudios del programa Water Law and Indigenous Rights (WALIR) en el área andina demuestran que el derecho al agua, vinculado siempre al derecho al territorio, es uno de los grandes retos que enfrentan los pueblos indígenas de América Latina (Gelles, 2002; Gentes, 2002; Guevara et al, 2002, entre otros). Se trata de un problema clave para el manejo del agua en el subcontinente, si se considera que cálculos conservadores estiman en 40 millones la población indígena en esta parte del mundo.

El objetivo de este trabajo es ofrecer un panorama general de los desafíos que encierra el manejo del agua por parte de los pueblos indígenas en México. La gestión democrática del agua debe incluir el respeto a los derechos de los pueblos indígenas y las comunidades campesinas y su inclusión efectiva en la toma de

\footnotetext{
${ }^{1}$ La presencia indígena en México va más allá de los grupos que hablan alguna de las lenguas americanas y se encuentra también en las costumbres y modos de vida de las comunidades rurales del país (Bonfil, 1987). Además, en un número muy alto de comunidades conviven indígenas y mestizos, y la toma de decisiones suele involucrarlos a todos, a través de las instituciones comunitarias. Por esa razón, a lo largo de este trabajo se hace mención a pueblos indígenas y comunidades campesinas.

${ }^{2}$ El resultado más importante de San Andrés, la iniciativa de ley indígena redactada por una comisión plural de legisladores, no fue aprobada por el Congreso. Los representantes del partido del presidente Fox (Partido Acción Nacional) fueron parte activa para modificar los términos de la iniciativa de ley convenida, que el presidente había prometido impulsar.
} 
decisiones. Desafortunadamente, este tema no ha recibido en México la atención debida por parte de las instituciones del Estado ${ }^{3}$.

El artículo está dividido en cuatro apartados. En el primero se ofrece un panorama general de los pueblos nativos de México y su relación con la disponibilidad de recursos hídricos. En el segundo se presentan algunos antecedentes sobre la gestión del agua en el país y el papel que ocupan en ella los indígenas.

El tercer apartado ofrece, de una manera resumida, algunos de los principales problemas que enfrentan las comunidades campesinas y los pueblos indígenas en la gestión del líquido. Se hace especial mención a los vínculos entre gestión del agua y manejo del territorio, un elemento central en el programa de reivindicaciones que han levantado diversas organizaciones indígenas de México. Finalmente se ofrecen algunas conclusiones y propuestas.

\section{Los Pueblos Indígenas de México: Un Breve Panorama}

México es un país de gran diversidad étnica y lingüística. Según el censo de población del 2000, existen un poco más de 10 millones de indígenas, 10.5 por ciento de la población total, que hablan más de 62 lenguas y viven en todo el país, aunque se concentran principalmente en los estados del centro y sur ${ }^{4}$.

Los estados con mayor población indígena son Oaxaca, con un millón y medio; Chiapas con un millón; Veracruz, Yucatán, Estado de México y Puebla, con alrededor de 900 mil indígenas cada uno.

Hidalgo, Guerrero, Quintana Roo, San Luis Potosí y Tabasco, son otros estados con una numerosa población indígena. Entre las entidades del norte, en la frontera con los Estados Unidos, Sonora tiene una importante población de Yaquis y Mayos, en Chihuahua viven los Tarahumaras y Coahuila cuenta con un pequeño grupo Kikapú. El 27 por ciento de los 2443 municipios del país tienen 40 por ciento o más de indígenas.

En el Distrito Federal, la capital mexicana, viven 333 mil de ellos, convirtiendo a la ciudad en la concentración urbana con el mayor número de indígenas en México. Las lenguas con mayor número de hablantes son el Náhuatl, el Maya y las diferentes variantes del Zapoteco y Mixteco.

La presencia de los pueblos indígenas no es un dato estadístico. Desempeñan un papel social muy importante en distintos aspectos de la vida del país y en particular tienen una intervención destacada en el manejo de los bosques, selvas y ambientes lacustres (Toledo y Argueta, 1992; Nigh y Rodríguez, 1995). La mayor parte de la superficie forestal del país es propiedad legal o está habitada por comunidades y pueblos indígenas, quienes se han convertido en actores destacados para la conservación del recurso forestal (Merino, 1997; Chapela, 1995).

\footnotetext{
${ }^{3}$ Llamar la atención sobre este punto y contribuir a la construcción de propuestas para que se respeten los derechos indígenas al agua, es uno de los objetivos fundamentales del programa Water Law and Indigenous Rights (WALIR), que desarrolla actividades de investigación, difusión y acompañamiento de organizaciones indígenas en varios países de América. Ver Boelens, 2002

${ }^{4}$ En México, como en otros países, se debate sobre cuál es la mejor forma de registrar la existencia de la población indígena. En los censos se ha optado por el criterio lingüístico. El Instituto Nacional Indigenista hizo una estimación global de 12 millones 707 mil indígenas para el año 2000. Para más datos, ver Serrano, et al, 2002.
} 
Los indígenas juegan también un papel importante en el aprovechamiento y protección de la biodiversidad y poseen un conocimiento amplio y complejo de los muy variados ecosistemas que habitan: el desierto, las planicies costeras, la selva húmeda y los bosques templados y fríos (Carabias et al, 1994).

En relación con el agua, la situación de los indígenas es heterogénea. La mayoría vive en la parte de México con mayor disponibilidad de agua, debido a que el centro y sobre todo el sur del país registra la precipitación pluvial media anual más alta. En algunas zonas de Chiapas, Oaxaca, Veracruz, Tabasco y San Luis Potosí, las lluvias alcanzan más de $3500 \mathrm{~mm}$. al año, frente al promedio nacional de $770 \mathrm{~mm}$.

En el otro extremo se encuentran pueblos que habitan zonas secas, como los otomíes (Hñähñü) del valle del Mezquital, donde llueven al año entre 350 y $400 \mathrm{~mm}$. en promedio, o los seris (Konkaak) de la costa de Sonora, con precipitaciones de entre 100 y $200 \mathrm{~mm}$. anuales.

La diversidad ambiental explica también las diferentes formas en que el agua es un elemento básico en la vida económica, social y cultural de estos pueblos. Para la mayoría es muy importante contar con el agua para sus cultivos, pero algunos se empeñan también en mantener en buen estado sus ríos, lagunas y esteros para pescar. Para todos es muy importante contar con el abastecimiento suficiente de agua de buena calidad para el consumo humano.

Es necesario subrayar que la relación de los pueblos indígenas con el agua no es de simples usufructuarios, sino que juegan -en particular las comunidades forestales- un papel muy importante en la conservación de las cuencas hidrográficas. En forma creciente las comunidades indígenas y campesinas se interesan por las condiciones generales en las que se maneja el territorio, para garantizar la cantidad y calidad del agua que requieren. Muchas comunidades indígenas forestales están comprometidas en un manejo sustentable del bosque, entre otras razones porque comprenden el papel que tiene para la cosecha de agua. En ese punto ofrecen servicios ambientales muy valiosos para la sociedad.

\section{La Gestión del Agua}

Los aprovechamientos del agua en México se rigen por el artículo 27 constitucional y su ley reglamentaria, la Ley de Aguas Nacionales, que entró en vigor el 2 de diciembre de $1992^{5}$. La definición de aguas nacionales en la ley es tan amplia, que prácticamente abarca todas las corrientes superficiales y regula la extracción del agua subterránea. El ejecutivo federal ejerce por ley, a través de la Comisión Nacional del Agua (CNA), la autoridad y administración en materia de los recursos hídricos del país.

El gobierno federal concentró el control de los recursos hídricos del país al término de un largo proceso que abarcó los últimos años del siglo XIX y todo el siglo XX. Desplazó a los gobiernos estatales y ayuntamientos del control de ríos, lagunas, manantiales y acuíferos. De la misma forma, los grupos de regantes y las autoridades comunitarias regidas por usos y costumbres, fueron perdiendo capacidades de decisión frente a la ingerencia federal (Sánchez, 1993; Aboites, 1998).

Este proceso se realizó gracias a diversas medidas jurídicas, políticas, técnicas y financieras, donde tuvo un papel muy importante la construcción de las grandes obras hidráulicas que cambiaron la configuración de muchas cuencas.

\footnotetext{
${ }^{5}$ En la actualidad está abierto un proceso para reformar la Ley de aguas Nacionales. Las modificaciones aprobadas por el Congreso fueron regresadas por el Presidente Fox, con una serie de observaciones que deben ser consideradas.
} 
La planeación y control de obras hidráulicas que modificaban las corrientes de agua a nivel de toda la cuenca, concentraron los conocimientos sobre el régimen hídrico en un grupo de expertos, los técnicos del gobierno federal, deslegitimando el conocimiento de los grupos locales. Al frente de esas obras, cuyo objetivo era ampliar la superficie irrigada, estuvo la Comisión Nacional de Irrigación y después la Secretaría de Recursos Hidráulicos.

La concentración del control de los recursos hídricos afectó de manera particular el derecho de los pueblos indígenas al aprovechamiento por usos y costumbres de lagunas, manantiales y ríos; también alteró los territorios donde se desecaron lagunas y humedales o se construyeron los grandes embalses, ocasionando modificaciones importantes en las formas de vida de innumerables comunidades.

El crecimiento significativo que experimentó la superficie irrigada del país entre 1920 y 1970 y el aumento en los volúmenes de energía eléctrica disponible para el crecimiento industrial y urbano, se consiguió con un modelo de gestión que concentró en manos del poder ejecutivo todas las decisiones del manejo del agua. En esas condiciones los proyectos de acondicionamiento hidráulico del territorio nacional alcanzaron magnitudes nunca antes vistas.

Hasta finales de la década de los ochenta del siglo XX, la participación social en la toma de decisiones fue inexistente. A los grupos afectados por una u otra decisión, solo les quedaba el camino de la protesta abierta.

Al amparo de estas disposiciones legales se ejecutaron obras como el trasvase de agua de la cuenca del río Lerma al Distrito Federal, afectando a muchas comunidades campesinas e indígenas del estado de México quienes vieron desaparecer las lagunas y humedales que utilizaban para la pesca y obtención de otros productos lacustres. Los pueblos dedicados a la agricultura fueron afectados por la pérdida de humedad para sus cultivos.

La Ley de 1992, a tono con los cambios experimentados en otros países, otorgó a la CNA la tarea de acreditar, promover y apoyar la organización de los usuarios "para mejorar el aprovechamiento del agua y la preservación y control de su calidad", e instauró la figura de consejo de cuenca como instancia de coordinación y concertación entre la CNA, los distintos niveles de gobierno y los usuarios, con el fin de mejorar la administración de las aguas.

La ley actual favorece la visión fragmentada en el manejo del agua, al enfocarse solo en los usos sectorizados (urbano, agrícola, industrial, energético) y dar muy poca atención a los impactos que esos usos tienen sobre los ecosistemas o sobre grandes territorios. En ese punto, la gestión actual del agua en México no ha cambiado mucho con respecto a la administración tradicional.

La conformación de los consejos de cuenca solo contempla la participación de los usuarios de acuerdo al tipo de uso del agua (agrícola, urbano, industrial), por lo que las comunidades y pueblos indígenas, interesados en los impactos que el manejo del agua puede tener en sus formas de vida y en general en el territorio que habitan, no tienen en esos organismos un espacio adecuado para expresarse e influir en la toma de decisiones ${ }^{6}$.

\footnotetext{
${ }^{6}$ En los últimos tres años se han empezado a incorporar a los consejos de cuenca representantes del "uso ambiental" del agua. Se trata de una formulación hecha para mantener la visión fragmentada, como si un lago o un estero fuera también "un usuario". Además, la representación generalmente se asigna a grupos ecologistas, sin considerar que autoridades municipales o dirigentes de organizaciones indígenas en algunas cuencas, podrían tener un interés más firme en el manejo integral del agua en un territorio.
} 
Por otra parte, la ley no reconoce a los pueblos indígenas derechos colectivos sobre el territorio que habitan, de tal manera que son escasos los recursos jurídicos que tienen a su alcance para detener las modificaciones irreversibles como los trasvases de agua y la construcción de presas ${ }^{7}$.

La formación de los consejos de cuenca es una disposición legal reciente (11 años), cuya puesta en práctica es débil y errática y no consigue cambiar el esquema de gestión caracterizado por la toma de decisiones concentradas en manos de los administradores federales, que actúan sin dar información ni consultar a los afectados.

Aunque en algunos foros internacionales México es reconocido por haber conseguido, en poco tiempo, la organización de consejos de cuenca que cubren prácticamente todo el país, en México muchas voces independientes han señalado que esos organismos en general se conforman con muy escasa legitimidad representativa, producto de la propia herencia corporativa y de clientelas políticas del régimen que dominó México durante los últimos 70 años.

La conformación y funcionamiento de los Consejos de Cuenca en México adolece de errores que lesionan su legitimidad y les impide funcionar como órganos realmente representativos de los diversos grupos interesados en la administración del agua. En esas condiciones, la gestión democrática del agua sigue siendo una tarea pendiente y es un reto construir formas de gestión que incluyan a los pueblos indígenas del país en la toma de decisiones.

\section{Impactos del Manejo del Agua sobre los Pueblos Indígenas}

Son muchos los terrenos en que el derecho al agua es difícil de ejercer para los pueblos indígenas. En el abasto para uso humano, un derecho básico, las localidades rurales enfrentan grandes carencias. Según estimaciones oficiales, el 42 por ciento de las viviendas indígenas de México carecían de agua entubada en el año 2000 y el 70 por ciento carecía de servicios de saneamiento ${ }^{8}$. Esto explica en parte el resurgimiento de enfermedades como cólera y la persistencia de la tifoidea en esas regiones.

México sigue registrando, particularmente en el medio rural, altos índices de enfermedades infecciosas cuya transmisión está asociada a la falta de agua o al consumo de aguas contaminadas. Los programas gubernamentales para enfrentar esta situación son débiles, de corto alcance y mal ejecutados.

Paradójicamente, existen planes para transferir el agua de regiones campesinas a las grandes ciudades, sin atender las mínimas necesidades de inversión para mejorar el abasto al consumo humano local. Es el caso de la transferencia de agua de la cuenca alta del río Balsas a la ciudad de México. En varios casos, las pequeñas comunidades campesinas e indígenas negocian la entrega de agua de sus manantiales para una ciudad, a cambio de que se les instale una red de abasto y distribución de agua.

Otro campo conflictivo es la contaminación de los recursos hídricos que utilizan los pueblos indígenas. Un caso extremo es la irrigación del valle del Mezquital con las aguas residuales de la ciudad de México. Esa región, habitada por comunidades campesinas e indígenas otomíes recibe, desde hace un siglo, el mayor

\footnotetext{
${ }^{7}$ Frente a casos semejantes, las comunidades indígenas pueden buscar amparo en la legislación ambiental del país, que ofrece pequeños resquicios para argumentar la falta de sustentabilidad de los proyectos que se pretenden ejecutar. También existen pequeños márgenes de intervención de las autoridades municipales para modificar algunos planes. Estos procedimientos dependen de una evaluación de impacto ambiental, que se ha revelado como un instrumento controvertido debido a que fácilmente son manipuladas por las empresas que las presentan.

${ }^{8}$ Contar con agua entubada no significa tener agua potable. En el medio rural, como en muchas ciudades de México, la potabilización del agua es un tema pendiente.
} 
volumen de aguas contaminadas que desaloja la capital del país. Los beneficios que los habitantes obtienen del riego de sus cultivos, no se comparan con los riesgos que implica para la salud el uso de aguas contaminadas (Peña, 1999). México tiene un gran rezago en el tratamiento de las aguas residuales y las regiones indígenas figuran entre las más abandonadas en ese aspecto.

Conviene detenernos con amplitud en uno de los impactos más importantes por la extensión de sus efectos en el tiempo y en el espacio: la relocalización forzada de la población indígena y campesina, debido a la construcción de grandes embalses para riego o generación de energía eléctrica.

El ejemplo más dramático y quizá el mejor documentado de esta política es lo que sucedió en la cuenca del río Papaloapan, en el istmo de Tehuantepec, con la construcción de las presas Miguel Alemán, con capacidad para 9 mil 106 millones de metros cúbicos y Cerro de Oro, con capacidad para 4 mil 400 millones de metros cúbicos. La primera se utiliza para generación de electricidad y control de avenidas y la segunda para riego agrícola y energía eléctrica.

Al inicio de los años cincuenta, la Comisión del Papaloapan puso en operación los planes para intervenir la cuenca del río. Para construir la presa Miguel Alemán se utilizaron $500 \mathrm{~km}^{2}$ del territorio del pueblo mazateco, un poco más de la quinta parte de la superficie en la cual vivían. Se expulsó de sus lugares de origen a 20 mil campesinos y aunque la presa se terminó en 1955, el reacomodo de las personas desplazadas concluyó muchos años más tarde, en 1962 (Barabas y Bartolomé, 1973; Boege, 1988; Nigh y Rodríguez, 1995).

Debido a que una buena parte de las poblaciones nativas ofrecieron resistencia a dejar sus tierras, el gobierno creó en 1954 el Centro Coordinador Indigenista de Temascal con el fin de convencer a los mazatecos afectados de la necesidad de la relocalización. La tarea principal de la oficina del Instituto Nacional Indigenista fue enfrentar las contingencias de organizar los nuevos pueblos. Veinte años después, el reacomodo masivo de indígenas se volvió a repetir para construir la presa Cerro de Oro, al suroriente de la primera, esta vez los desplazados fueron principalmente indígenas chinantecos.

En el caso de Cerro de Oro, la expulsión de los indígenas se acompañó de un programa para la colonización de tierras de la selva tropical, abiertas al cultivo irrigado. Unos 13 mil chinantecos desplazados por la presa fueron llevados al Uxpanapa, que se acondicionó en forma apresurada como distrito de riego, con la inversión de 50 millones de dólares otorgados por el Banco Interamericano de Desarrollo (BID).

Tanto los mazatecos como los chinantecos expulsados de sus tierras, tardaron en reconstruir los vínculos comunitarios que se vieron desgarrados con el éxodo. En la reubicación perdieron la comunicación cotidiana con una buena parte de sus familiares y vecinos anteriores; también fueron despojados de símbolos de identidad muy importantes como sus sitios sagrados y las tumbas de sus antepasados. Las familias que se opusieron hasta el último momento al desalojo, fueron removidas con el uso de la fuerza pública.

Pronto se descubrió que los terrenos ganados a la selva no eran los mejores para el monocultivo de arroz y pastizales. En pocos años se perdieron los suelos frágiles y la introducción de ganado bovino terminó de degradar las antiguas tierras de selva húmeda (Toledo, 1984).

El compromiso gubernamental de ofrecer mejores condiciones de vida a los pueblos desplazados, nunca se cumplió. Las nuevas localidades no tenían servicios públicos básicos en un 50 por ciento y tampoco fueron consistentes los apoyos para capacitación e inversión agrícola. 
La construcción de las presas Miguel Alemán y Cerro de Oro es, por diversos motivos, un ejemplo de lo que no se debe hacer si se quiere respetar y proteger los derechos de los pueblos indígenas, a los que nunca se tomó en cuenta en aquellas decisiones.

El reacomodo forzado de pueblos indígenas por la construcción de embalses es uno de los temas más sensibles en México. Por esa razón, ha despertado especial inquietud la reactivación por el gobierno del presidente Fox de la construcción de un sistema de presas sobre la cuenca del río Usumacinta, en la frontera con Guatemala. La más importante de ellas será la presa de Boca del Cerro, un proyecto binacional México-Guatemala, realizado en el marco del Plan Puebla Panamá impulsado por el presidente Fox.

La cuenca del río Usumacinta abarca la región de los altos de Chiapas, la selva Lacandona y una parte del estado de Tabasco en México y los departamentos de El Quiché, Verapaz y El Petén en Guatemala. Claramente se trata de territorios indígenas. Para el caso de Chiapas, en esa región se concentran los municipios con 40 por ciento o más de población indígena.

La cuenca del Usumacinta es una de las regiones con mayor diversidad biológica y cultural, en donde se localizan muchos sitios arqueológicos de la cultura maya. Es también una región con importantes yacimientos de petróleo.

Según los planes de la Comisión Federal de Electricidad el proyecto de Boca del Cerro se construirá a 9.5 kilómetros al suroeste de la ciudad de Tenosique, en Tabasco. La cortina de 135 metros de altura estaría en el lado mexicano y represaría 30 mil 500 millones de $\mathrm{m}^{3}$, la mitad en Guatemala y la otra en México, aunque el 65 por ciento de los aportes de agua serán de los afluentes guatemaltecos (México Tercer Milenio, 2003).

Según los impulsores del plan, el área inundada será de $1645 \mathrm{~km}^{2}, 530$ en México y 115 en Guatemala. La pretensión es generar 17 mil 400 millones de kilowatts-hora. Afirman que la obra significaría un gran ahorro de combustibles (29 millones de barriles de combustóleo por año) y una aportación de energía indispensable para el crecimiento económico de México y sus vecinos.

La presa Boca del Cerro es parte de un plan de grandes transformaciones en el régimen hídrico de la cuenca del Usumacinta que incluye la construcción del canal de derivación Balancán, para trasvasar caudales del río a la Laguna de Términos, que se aprovecharán para la generación de 1250 millones de kilowatts-hora adicionales.

Con la construcción de la presa Salto del Agua y un amplio sistema de drenaje, el plan del gobierno federal planea disponer también de un millón y medio de hectáreas para cultivos, ganadería y actividades acuícolas.

En junio del 2002, un diario de Tabasco publicó el "Memorándum de Cooperación Eléctrica con Centroamérica" que Fox había firmado días antes. Pocas semanas después, el mismo diario hizo público una parte del proyecto ${ }^{9}$. El director de la Comisión Federal de Electricidad confirmó la construcción de la presa, al indicar que no sería de cortina alta, sino baja, para disminuir el área inundada. Sin embargo, hasta

${ }^{9}$ Ver Tabasco Hoy, 30 de junio y 10 de agosto del 2002. La información se publicó también en otros diarios, ver New York Times del 22 de septiembre de 2002 
octubre del 2003, las autoridades no han ofrecido información completa de los planes que se quieren ejecutar $^{10}$.

Organismos no gubernamentales, organizaciones indígenas, ecologistas, intelectuales y artistas, han manifestado fuertes críticas al proyecto ${ }^{11}$. Subrayan principalmente el impacto negativo que el embalse tendría en las poblaciones indígenas y campesinas, que serán desplazadas de la superficie inundable. Según cálculos moderados, entre 30 mil y 50 mil personas deberán abandonar sus hogares y tierras de cultivo, lo que significa un éxodo semejante al impuesto en los casos de las presas Cerro de Oro y Miguel Alemán ${ }^{12}$.

Por la experiencia de casos anteriores semejantes, es posible prever que los campesinos e indígenas desplazados no serán reubicados en mejores condiciones sino que, por el contrario, los espera un futuro aún más precario.

Los embalses también atentan contra el patrimonio cultural de los pueblos nativos, amenazando algunos sitios arqueológicos de la cultura maya como Yaxchilán en Chiapas y Piedras Negras en El Petén guatemalteco. La biodiversidad de la región también se encuentra amenazada. Bajo el agua se perderían los nichos ecológicos de diversas especies animales y vegetales, mientras en otras partes se desecarían humedales como los pantanos de Centla.

La coalición de organismos y personalidades opositoras al proyecto, en el que participan activamente varias organizaciones y ayuntamientos indígenas, ha llamado la atención sobre los poderosos intereses económicos que están involucrados. La reactivación del plan para construir el sistema de presas sobre el Usumacinta tiene lugar mientras el presidente Fox insiste en modificar la Constitución del país para permitir la inversión privada en el sector eléctrico, sobre todo por parte de compañías transnacionales como Enrón, que tendrían una plataforma excelente para vender electricidad hacia Centroamérica.

En el plan también están interesadas las grandes compañías de constructores con vínculos muy fuertes con la administración federal, que con frecuencia han sido denunciadas de prácticas corruptas en la asignación de contratos. Se calcula que la presa Boca del Cerro costaría 5 mil millones de dólares, por lo que los constructores tienen a la vista un negocio muy importante.

Los planes gubernamentales en marcha y el movimiento ciudadano que se opone a la construcción de estos embalses, representan un reto para el consejo de cuenca. ¿Es un organismo realmente existente y representativo? ¿Podrá funcionar como foro de información y consulta de los afectados?. Todo parece indicar que no. Las críticas de los organismos ciudadanos y los desmentidos de las autoridades, han circulado al margen del que por ley debería ser el principal organismo de información, consulta y consenso.

En particular, los organismos indígenas y campesinos de la zona que han manifestado distintas inquietudes, están siendo ignorados. El gobierno federal está respondiendo de la misma manera como lo hacían los gobiernos anteriores.

\footnotetext{
${ }^{10}$ La Comisión Nacional del Agua no ha publicado los detalles del proyecto completo, aunque se informó que la Comisión Federal de Electricidad inició las obras en marzo de este año.

${ }^{11}$ Marzo 2002, declaración del Foro Mesoamericano contra las Represas.

12 Otros aseguran que los desplazados pueden llegar a un millón de personas por el proyecto completo de obras, (Tabasco Hoy, 26 de enero, 2003).
} 


\section{Conclusiones}

En México, el respeto a los derechos indígenas al agua y su inclusión en la gestión del líquido, son temas pendientes. Pese a la importancia que las comunidades nativas tienen y pueden tener en la conservación de las cuencas, el espacio clave para la conservación de los recursos hídricos, la voz indígena es débil en los organismos de gestión.

El diseño de representaciones por uso del agua, que domina los consejos de cuenca, impide que tenga cabida en ellos la voz de los indígenas, interesados en temas como la protección de sus territorios comunitarios y el manejo de los recursos hídricos no sectorizados. El manejo del agua y sus relaciones con la conservación de la biodiversidad y el patrimonio cultural, son temas de interés para los pueblos indígenas que no pueden reducirse al interés de usuarios agrícolas o urbanos.

Los principales retos que los pueblos indígenas enfrentan están asociados, por una parte, a la fragilidad de los recursos jurídicos que tienen a su alcance para ser reconocidos como sujetos de derecho sobre un territorio. Con el incumplimiento de los acuerdos de San Andrés, las comunidades nativas están obligadas a buscar en la legislación ambiental y en otros ordenamientos legales, algunos recursos para detener las modificaciones hídricas que atentan contra ellos.

La fragilidad de los recursos jurídicos a su alcance, se suma a la fragilidad social en que se encuentran cuando deben enfrentar intereses económicos muy poderosos, como los que están en juego en la construcción de embalses para electricidad.

Para garantizar el respeto a los derechos indígenas al agua, incluido el respeto a que no se les despoje de sus lugares de vida, es necesario trabajar en tres direcciones: la insistencia en la adopción de un marco jurídico que reconozca plenos derechos a los pueblos nativos; la inclusión de las representaciones legítimas de los indígenas en los órganos de gestión del agua, en los territorios donde viven; y la formación de coaliciones sociales que amplifiquen la voz de los ciudadanos que, como los indígenas, generalmente se encuentran en desventaja frente a los intereses financieros más poderosos.

\section{Referencias}

Aboites, Luis. 1998. El agua de la nación. Una historia política de México (1888-1946). México: CIESAS.

Barabas, Alicia y Miguel Bartolomé. 1973. "Hydraulic development and ethnocide. The mazatec and chinantec people of Oaxaca". En: International Workgroup for Indigenous Affairs, num 15, Copenhague

Boege, Eckart. 1988. Los mazatecos ante la nación. Contradicciones de la identidad étnica en el México actual. México: Siglo XXI.

Boelens, Rutgerd. 2002. "Water Law and Indigenous Rights: Research, action and Debate". En: WALIR studies, Volume 2, Wageningen University/ IWE and United Nations/CEPAL, Wageningen.

Bonfil, Guillermo. 1987. México Profundo: una civilización negada. México: CIESAS/SEP.

Carabias, Julia, Enrique Provencio y Carlos Toledo. 1994. Manejo de recursos naturales y pobreza rural. México: UNAM-FCE. 
Chapela, Gonzalo. 1995. Aprovechamiento de los recursos forestales en la Sierra Purépecha. México: UAM.

Gelles, Paul. 2002. "Andean Cultura, Peasant Communities and Indigenous Identity". En: WALIR studies, Volume 2, Wageningen University/ IWE and United Nations/CEPAL, Wageningen.

Gentes, Ingo. 2002. "Water Law and Indigenous Rights in the Andean Countries: conceptual elements". En: WALIR studies, Volume 2, Wageningen University/ IWE and United Nations/CEPAL, Wageningen. Guevara-Gil, Armando. et al. 2002. "Water Legislation and Indigenous Water Management in Peru". En: WALIR studies, Volume 2, Wageningen University/ IWE and United Nations/CEPAL, Wageningen.

Merino, Leticia (coordinadora). 1997. El manejo forestal comunitario en México y sus perspectivas de sustentabilidad. México: UNAM, SEMARNAP, WRI, CMS.

Nigh, Ronald y Nemesio J. Rodríguez. 1995. Territorios violados. México: INI-CNCA.

Peña, Francisco. 1999. "La esperanza en las aguas de desecho, Construcción de una región irrigada en el valle del Mezquital. En: Frontera Interior, revista de ciencias sociales y humanidades, Num. 3-4, INAHUniversidad de Querétaro, Querétaro.

Sánchez, Martín. 1993. "La herencia del pasado. La centralización de los recursos acuíferos durante el porfiriato 1888-1910". En: Relaciones. Estudios de Historia y Sociedad, No. 54, El Colegio de Michoacán, Zamora.

Serrano, Enrique, Arnulfo Embriz y Patricia Fernández (coordinadores). 2002. Indicadores socioeconómicos de los pueblos indígenas de México. México: INI, Programa de Naciones Unidas para el Desarrollo, CONAPO.

Toledo, Alejandro. 1984. Cómo destruir el paraíso: el desastre ecológico del sureste. México: OcéanoCecodes.

Toledo, Víctor y Arturo Argueta. 1992. “Cultura indígena y ecología”. En: Plan Pátzcuaro 2000. MéxicO. Fundación Friedrich Ebert. 